\title{
\begin{tabular}{l|l} 
Mibraries & DSpace@MIT
\end{tabular}
}

\author{
MIT Open Access Articles
}

The application of HPLC/MS analysis with a multienzyme digest strategy to characterize different interferon product variants produced from Pichia pastoris

The MIT Faculty has made this article openly available. Please share how this access benefits you. Your story matters.

As Published: https://doi.org/10.1007/s00726-019-02772-9

Publisher: Springer Vienna

Persistent URL: https://hdl.handle.net/1721.1/131809

Version: Author's final manuscript: final author's manuscript post peer review, without publisher's formatting or copy editing

Terms of Use: Article is made available in accordance with the publisher's policy and may be subject to US copyright law. Please refer to the publisher's site for terms of use. 


\section{The application of HPLC/MS analysis with a multi-enzyme digest strategy to characterize different interferon product variants pro- duced from Pichia pastoris}

Cite this article as: Yu Wang, Di Liu, Laura E. Crowell, Kerry R. Love, Shiaw-lin Wu and William S. Hancock, The application of HPLC/MS analysis with a multi-enzyme digest strategy to characterize different interferon product variants produced from Pichia pastoris, Amino Acids https://doi.org/10.1007/s00726-019-02772-9

This Author Accepted Manuscript is a PDF file of an unedited peer-reviewed manuscript that has been accepted for publication but has not been copyedited or corrected. The official version of record that is published in the journal is kept up to date and so may therefore differ from this version.

Terms of use and reuse: academic research for non-commercial purposes, see here for full terms. https://www.springer.com/aam-terms-v1 


\section{The application of HPLC/MS analysis with a multi enzyme} digest strategy to characterize different interferon product variants produced from Pichia Pastoris

Yu (Annie) Wang ${ }^{1}$, Di Liu ${ }^{2}$, Laura E. Crowell ${ }^{2}$, Kerry R. Love ${ }^{2}$, Shiaw-lin Wu ${ }^{3}$, William S. Hancock $^{1^{*}}$

1 Barnett Institute and Department of Chemistry and Chemical Biology, Northeastern University, 360 Huntington Avenue, Boston, Massachusetts 02115, United States

2 Koch Institute for Integrative Cancer Research, Massachusetts Institute of Technology, 500 Main

Street, Cambridge, Massachusetts, 02142, United States

3 BioAnalytix Inc., 790 Memorial Drive, Cambridge, Massachusetts 02139, United States

*Corresponding Author

wi.hancock@northeastern.edu

Abstract:

Interferons are signaling proteins that belong to the large class of cytokines and human interferons which are classified based on the type of receptor interactions: type I, II and III. IFNa2b belongs to the type I interferon class with a major therapeutic application for the treatment of hepatitis B and C infections. A recombinant form of IFNa2b expressed in E.coli, known as IntronA, has been approved by US Food and Drug Administration (FDA). IFNy, also known as type II interferon, plays a significant role in the inhibition of viral replication. Actimmune ${ }^{\circledR}$ is a US Food and Drug Administration (FDA) approved version of IFNy for the indication of reducing infections associated with Chronic Granulomatous Disease and Severe Malignant Osteopetrosis. In this study we have 
applied advanced analytical methods for the characterization of IFNa2b and IFNy produced from Pichia pastoris. The multi-enzyme digestion approach has been developed to allow measurement of $100 \%$ sequence coverage and detailed analysis of post-translational variants and degradation products. In this manner we identified the following variants in IFN $a 2 b$ : N-terminal residual leader sequence, an amino acid substitution, oxidation of methionine residues and two sites of high-mannose $\mathrm{N}$ glycosylation. In the Pichia IFN y produced material our approach detected variants resulting from glycosylation, C-terminal proteolysis, oxidation of methionine residues and deamidation.

In this manner the analytical program was able to support rapid process development as well as identify product variants and degradation products in the resulting product.

\section{Introduction:}

Human interferon $\alpha 2 b$ is a subtype of the human alpha interferon family and its potential therapeutic applications have been investigated extensively. ${ }^{1,2}$ Recombinant IFN $\alpha 2 b$ is a 165-amino acid single chain polypeptide with a molecular weight $19.2 \mathrm{KDa}$. Licensed products include Intron A, first marketed by Schering Corp (Merck) in $1986,{ }^{3}$ using $E$. coli expression as well as Shanferon, sold by Shantha in India. and manufactured in Pichia pastoris. Intron A is widely used for the treatment of myelomas, lymphomas, solid tumors and chronic hepatitis B and C infections. Interferon gamma (IFN $\mathrm{Y}$ ) is the single member of the type II class of interferons and it is produced by lymphocytes activated by specific antigens. In addition to antiviral activity, IFN $\mathrm{Y}$ has important immunoregulatory functions. ${ }^{4}$ Interferon gamma-1b, marketed as Actimmune ${ }^{\circledR}$, is a 143-amino acid protein and is produced in $E$. coli with an additional N-terminal 
methionine residue. Unlike human IFN gamma, Actimmune is not glycosylated as a result of using $E$. coli as the host system.

Nowadays, there is a growing interest in the development of biosimilars which can provide patients affordable drugs and also bring financial benefits to the biopharmaceutical industry due to increased patient access. In the case of interferon biosimilars there are commercial opportunities as the patents for both Intron A (IFN $\alpha 2 b$ ) and Actimmune (IFN Y) have expired. In fact, development of interferon biosimilars are now underway in many biopharmaceutical companies around the world. For example, Biosidus, Pharmaclon and Zydus are all developing biosimilars of IFN $\alpha 2 b .{ }^{5}$ In a development and regulatory pathway, obtaining analytical data is the primary step to prove the similarity between the reference material and biosimilars. Due to the higher complexity of biosimilars than small molecules, new analytical methods need to be developed to support the comparability of the biosimilar product to the reference standard.

In the biopharmaceutical industry, there has been a growing interest in using Pichia Pastoris as a host system due to favorable factors such as high growth rates which allows reduced fermentation times, the use of defined instead of complex media and secretion of low levels of host cell proteins. The manufacture of therapeutics in yeast has been validated by product approvals by both the US Food and Drug Administration (FDA) and the European Medicines Agency (EMA). In engineered strains yeast is capable for production of human-like post-translational modifications. ${ }^{6}$ All of these factors act to lower the barrier to effective development of lower price therapeutics. In this program we used codon-modified Pichia pastoris cloned into a pPICZa-family factor 
that was fused with a truncated form of the Saccharomyces cerevisae $\alpha$-factor secretion signal, under control of the methanol-inducible AOX1 promoter. The Saccharomyces cerevisae $\alpha$-factor preprosequence is one of the most widely used secretory signal in Pichia pastoris, but one concern that can arise is the generation of $\mathrm{N}$-terminal variant(s) due to the incomplete removal of the signal peptide. It has been reported that during the biosynthetic process this sequence is cleaved into three peptides (one very long) upon secretion. ${ }^{7}$ The endogenous enzymes responsible for these cleavage events are Kex2 and Ste13 and the action of these enzymes generates heterogeneity in the $\mathrm{N}$-terminal amino acid sequence of the secreted product due to the incomplete removal of leader sequence. ${ }^{8}$ For example, the most common variant observed contains an additional EAEA tetrapeptide at the N-terminus. ${ }^{9}$ However, the a factor vector can be designed without the EAEA repeats to minimize the amount of $\mathrm{N}$-terminal heterogeneity. ${ }^{10} \mathrm{~A}$ further limitation with the use of Pichia Pastoris is that N-linked glycosylation is restricted to the high-mannose type, which is immunogenic ${ }^{11}$ and results in poor pharmacokinetic parameters due to rapid clearance via binding to high mannose receptors. $^{12}$

Interferon expression in Pichia Pastoris has previously been attempted however, with little protein characterization or product quality information. ${ }^{13}$ This paper presents the use of advanced analytical methods for the in-depth characterization of interferon products to produce in Pichia Pastoris. We used the multi-enzyme digestion strategy coupled with liquid chromatography - mass spectrometry analysis ${ }^{14,15}$ for the identification of product variants of interferons produced in the fermentation studies, 
including residual leader sequence, an amino acid substitution, backbone cleavages, glycosylation as well as degradation reactions.

\section{Methods and materials:}

\section{Reagents:}

Ammonium bicarbonate, dithiothreitol and iodacetamide was from Sigma Aldrich. Trypsin, and GluC was purchased from Promega. Guanidine hydrochloride, formic acid optima LC/MS grade, water LC/MS grade, acetonitrile (ACN), Methanol, Sulphosalicylic acid and trichloroacetic acid, hydrochloric acid was purchased from Fisher Scientific. NuPAGE Novex 4-12\% Bis-Tris gel, $1.0 \mathrm{~mm}, 10$ well, NuPAGE MES SDS running buffer (20X), NuPAGE LDS Sample Buffer (4X), PageRuler Prestained 10-180 KDA Protein Ladder, Invitrogen SimpleBlue SafeStain, Novex pH 3-10 IEF Protein Gels, 1.0 mm, 12 well, Novex pH 3-10 Buffer Kit (includes Novex IEF Anode Buffer 50X, Novex IEF Cathode Buffer pH 3-10 10X and Novex IEF Sample Buffer pH 3-10 2X), Colloidal Blue Staining Kit and premium grade TCEP-HCl were purchased from Thermo Fisher Scientific. IEF standards was from Bio Rad. IFN y standard material was purchased from Abcam (Cambridge, MA).

\section{Equipment:}

XCell SureLock Mini-Cell electrophoresis apparatus was from Thermo Fisher Scientific. A benchtop centrifuge was purchased from Corning. Ultimate 3000 nano LC pump (Dionex, Mountain View, CA). $10 \mu \mathrm{m}$ ID emitter (New Objective, Woburn, MA), LTQ-XL mass spectrometer (Thermo Fisher Scientific, San Jose, CA).

\section{SDS-PAGE of interferon $\alpha 2 b$ :}


$500 \mathrm{~mL} 1 \mathrm{X}$ NuPAGE SDS running buffer was prepared by mixing $25 \mathrm{~mL}$ MES running buffer with $475 \mathrm{~mL}$ deionized water. $10 \mu \mathrm{l}(1 \mu \mathrm{g} / \mu \mathrm{l})$ IFN $\alpha 2 \mathrm{~b}$ standard with $3.3 \mu \mathrm{l}$ LDS sample $(4 \mathrm{x})$ were mixed and $10 \mu \mathrm{l}(1 \mu \mathrm{g} / \mu \mathrm{l})$ Pichia IFN with $3.3 \mu \mathrm{l}$ LDS sample buffer were mixed. Gel apparatus was set and $5 \mu$ l protein ladder was loaded in lane 1 , one blank lane was left between each sample and IFN standard and Pichia IFN were loaded. The voltage was set at $160 \mathrm{~V}$ and run for $50 \mathrm{~min}$. Gel apparatus was disconnected and the gel was removed from the two plates, stained with SimpleBlue SafeStain for 30min and destained in deionized water overnight.

\section{Isoelectric focusing (IEF) of interferon $\alpha \mathbf{2 b}$ :}

$200 \mathrm{ml}$ cathode buffer for upper chamber was prepared by mixing $20 \mathrm{~mL}$ cathode buffer with $180 \mathrm{~mL}$ deionized water. $500 \mathrm{~mL}$ anode buffer for lower chamber was prepared by mixing $10 \mathrm{~mL}$ anode buffer with $490 \mathrm{~mL}$ deionized water. $10 \mu \mathrm{I}$ IFN standard was mixed with $10 \mu \mathrm{l}$ IEF sample buffer, same as Pichia IFN. Gel apparatus was set and $200 \mathrm{ml}$ cathode buffer was filled in the upper chamber and $500 \mathrm{ml}$ anode buffer was filled in the outer chamber. $2 \mu \mathrm{IIEF}$ standard was loaded on lane 1, one blank lane was left on purpose to avoid the contamination of the samples to each other. IFN standard and Pichia IFN were loaded. The whole apparatus was put in an ice bucket. The voltage program was set as follows: $100 \mathrm{~V}$ for $60 \mathrm{~min}, 200 \mathrm{~V}$ for $60 \mathrm{~min}$ and $500 \mathrm{~V}$ for $30 \mathrm{~min}$. While in the last $30 \mathrm{~min}$ of the run, fixing solution was prepared by adding $11.46 \mathrm{~g}$ trichloroacetic acid and $4.6 \mathrm{~g}$ sulphosalicylic acid in $90 \mathrm{ml}$ water. When the voltage program finished, the gel was washed with deionized water three times and then transferred to the fixing solution. The gel was fixed for $60 \mathrm{~min}$ on a shaker. Staining solution was prepared by mixing $58 \mathrm{~mL}$ water, $20 \mathrm{~mL}$ methanol, $20 \mathrm{~mL}$ stainer $\mathrm{A}$ and 2 
$\mathrm{mL}$ stainer $\mathrm{B}$ of the colloidal staining kit. The gel was then destained in water for overnight.

\section{Trypsin digestion of interferon $\alpha \mathbf{2 b}$ :}

$20 \mu \mathrm{L} 6 \mathrm{M}$ guanidine hydrochloride (MW $95.53 \mathrm{~g} / \mathrm{mol}$ ) in $0.1 \mathrm{M} \mathrm{NH} 4 \mathrm{HCO} 3$ was added to $20 \mu \mathrm{g}$ IFN $\alpha 2 \mathrm{~b}$ reference material and Pichia IFN $\alpha 2 \mathrm{~b}$. Followed by adding $2 \mu \mathrm{L}$ of $1 \mathrm{M}$ Dithiothreitol (DTT) solution to the solution (final concentration of DTT: $5 \mathrm{mM}$ ) and heating at $37^{\circ} \mathrm{C}$ for 30 minutes. Fresh $1 \mathrm{M}$ iodacetamide (IAA) solution was made in a foil-wrapped tube to avoid exposure to light and was added to the denatured, reduced IFN solution to final concentration of $10 \mathrm{mM}$. The tube was covered with aluminum foil and incubated at room temperature $\left(25^{\circ} \mathrm{C}\right)$ for $1 \mathrm{hr}$. After incubation, the solution was buffer exchanged to $100 \mathrm{mM}$ ammonium bicarbonate pH8 using 10 KDA cutoff spin column with speed of $14000 \mathrm{rpm}$ for $6 \mathrm{~min}$. For buffer exchange, $100 \mathrm{mM}$ ammonium bicarbonate solution was added with final volume $500 \mu \mathrm{l}$ in the cut-off tube and reduced to $100 \mu$ leach time. This step was repeated three times to ensure $\mathrm{pH} 8$ solution for enzyme digestion. An initial aliquot of trypsin $(1 \mu \mathrm{l}$ at $0.5 \mu \mathrm{g} / \mu \mathrm{l})$ was added to the solution and incubated at $37^{\circ} \mathrm{C}$ for 4 hours. Then a second aliquot of the same amount of enzyme was added for incubation overnight $(16 \mathrm{~h})$ at room temperature. The digestion was stopped by adding $4 \mu \mathrm{l} 1 \%$ formic acid to final $\mathrm{pH}$ around 3 . The peptide solution was aliquoted to a $30 \mu \mathrm{l} /$ vial and stored at $-80^{\circ} \mathrm{C}$ before $\mathrm{LC} / \mathrm{MS}$ analysis.

\section{GluC digestion of interferon $\alpha 2 b$ :}

$20 \mu \mathrm{L} 6 \mathrm{M}$ guanidine hydrochloride (MW $95.53 \mathrm{~g} / \mathrm{mol}$ ) $0.1 \mathrm{M}$ ammonium bicarbonate was added to $20 \mu \mathrm{g}$ IFN $\alpha 2 \mathrm{~b}$ reference material and Pichia IFN $\alpha 2 b$. Followed by adding 2 $\mu \mathrm{L}$ of $1 \mathrm{M}$ Dithiothreitol (DTT) solution to the solution (final concentration: $5 \mathrm{mM}$ DTT) 
and heated at $37^{\circ} \mathrm{C}$ for 30 minutes. Fresh $1 \mathrm{M}$ iodacetamide (IAA) solution was made in a foil-wrapped tube to avoid exposure to light and was added to the denatured, reduced IFN solution to final concentration of $10 \mathrm{mM}$. The tube was covered with aluminum foil and incubated at room temperature $\left(25^{\circ} \mathrm{C}\right)$ for $1 \mathrm{hr}$. After incubation, the solution was buffer exchanged to $100 \mathrm{mM}$ ammonium bicarbonate $\mathrm{pH} 8$ using 10 KDA cutoff spin column with speed of $14000 \mathrm{rpm}$ for $6 \mathrm{~min}$. For buffer exchange, $100 \mathrm{mM}$ ammonium bicarbonate solution was added to final volume $500 \mu \mathrm{l}$ in the cut-off tube and reduced to $100 \mu \mathrm{l}$ each time. This step was repeated three times to achieve $\mathrm{pH} 8$ solution for enzyme digestion. First aliquot of GluC $(1 \mu \mathrm{l}$ at $0.5 \mu \mathrm{g} / \mu \mathrm{l})$ was added to the solution and incubated at $37^{\circ} \mathrm{C}$ for $4 \mathrm{~h}$. Then a second dose of the same amount of enzyme was used for incubation overnight (16 h at room temperature. The digestion was stopped by adding $4 \mu \mathrm{l} 1 \%$ formic acid to make the final $\mathrm{pH}$. The peptide solution was aliquoted to a $30 \mu \mathrm{l} /$ vial and stored at $-80^{\circ} \mathrm{C}$ before LC/MS analysis.

\section{In-gel digestion of IFN Y gel:}

Day 1: The gel bands were cut into small pieces using a scalpel. $200 \mu \mathrm{l}$ of acetonitrile (ACN) and LC-MS water (1:1) was added to cover the gel pieces and shake at $800 \mathrm{rpm}$ on Eppendorf incubator for 1 hour until the gel pieces are clear. The liquid was removed. $100 \mu \mathrm{l}$ of acetonitrile was added to shrink the gel pieces and the liquid was removed afterwards. The gel pieces were then rehydrated with trypsin solution (12.5 ng/ $\mu \mathrm{l}$ trypsin in $50 \mathrm{mM}$ ammonium bicarbonate) at $4{ }^{\circ} \mathrm{C}$ for $30 \mathrm{~min}$. After rehydration, the remaining supernatant was removed, $100 \mu \mathrm{l} 50 \mathrm{mM}$ ammonium bicarbonate was added to cover the gel pieces followed by incubation at $37{ }^{\circ} \mathrm{C}$ overnight. Day 2: $50 \mu \mathrm{l} 25 \mathrm{mM}$ ammonium bicarbonate was added to the gel pieces and incubated at $37^{\circ} \mathrm{C}$ for $15 \mathrm{~min}$ 
with shaking. Liquid was spun down and $100 \mu \mathrm{l}$ acetonitrile was added followed by incubation at $37{ }^{\circ} \mathrm{C}$ for $15 \mathrm{~min}$ with shaking. The supernatant was spun down and collected in another tube. The extraction steps were repeated 3 times. In the last extraction step, $50 \mu \mathrm{l} 1 \%$ formic acid was added to stop the digestion. The combined extracts were concentrated to $10 \mu \mathrm{l}$ using a speed vacuum concentrator to around $10 \mu \mathrm{l}$.

\section{LC-MS/MS:}

An Ultimate 3000 nano LC pump (Dionex, Mountain View, CA) and self-packed C18 column (Magic C18, 200Å pore and $5 \mu \mathrm{m}$ particle size, $75 \mu \mathrm{m}$ internal diameter (ID) by $100 \mathrm{~mm}$ ) connected to a coated $10 \mu \mathrm{m}$ ID emitter (New Objective, Woburn, MA) were coupled online to an LTQ-XL mass spectrometer (Thermo Fisher Scientific, San Jose, CA) through a nanospray ion source (New Objective, Woburn, MA). Mobile phase A was $0.1 \%$ formic acid in HPLC grade water and mobile phase B was $0.1 \%$ formic acid in acetonitrile. The flow rate was $250 \mathrm{~nL} / \mathrm{min}$ with $2 \% \mathrm{~B}$ for $25 \mathrm{~min}$. The flow rate was maintained at $200 \mathrm{nl} / \mathrm{min}$ during the separation. The gradient is as follows: $0-60 \mathrm{~min} 2-$ $40 \% \mathrm{~B}, 60-70 \min 40-90 \% \mathrm{~B}, 70-75 \mathrm{~min}$ isocratic at $90 \% \mathrm{~B}$ and $75-78 \mathrm{~min} 2 \% \mathrm{~B}$. The mass spectrometer was operated in a data dependent mode (DDA) to switch between MS and CIDMS2. Briefly, after a full-scan MS spectrum from m/z 400-2000 in the linear ion-trap, 8 CIDMS2, with $28 \%$ normalized collision energy and activation $Q$ at 25 , activation steps were performed on the 8 most intense precursor ions from the full scan.

\section{Results:}

Detailed analytical information is critical for process development at an early stage where prompt analytical feedback will aid optimal strain development and high yield fermentation conditions, as well as characterization of the final product and the 
demonstration of biosimilarity. The examination of two related but quite different interferons allowed the exploration of the development of analytical methods suitable for the identification and characterization of a range of variants that are produced in the two processes. In the case of Pichia IFN $\alpha 2 b$, incomplete removal of $\mathrm{N}$-terminal signal peptide lead to $\mathrm{N}$-terminal variants, the presence of methionine residues results in the potential for oxidation, amino acid substitution was also observed. For Pichia IFN $\mathrm{y}$, the glycosylation motifs N-X-S/T, N98 (NYS) and N26 (NGT) in the amino acid sequence can lead to a high level of glycosylation. The similarity of the amino acid sequence in the C-terminus and the signal peptide can be the cause of C-terminal proteolysis, as the enzyme responsible for removal of signal peptide can also recognize the amino acids near the C-terminus. Methionine and asparagine residues have the potential of oxidation and deamidation.

\section{Part A: Analysis of Interferon (IFN) $\alpha 2 b$ reference material and Pichia IFN $\alpha 2 b$}

\section{SDS-PAGE of IFNa2b}

Molecular weight determination by SDS-PAGE can provide information about the presence of product variants such as residual leader sequence and proteolytic processing, as well as a comparison between the process sample and a reference material. As shown in Figure S1, both IFN $\alpha 2 b$ reference material and Pichia $\alpha 2 b$ have the molecular weight of approximately $19 \mathrm{KDA}$ and a single band on SDS-PAGE. For an in-depth comparison of standard and the Pichia-derived material, LC/MS analysis was then performed on the digest of the two samples (see later).

\section{IEF gel of IFNa2b}


Isoelectric focusing can be used to detect charge variants present in a process development sample such as deamidation and changes in the degree of sialyation. In Figure 1 we compared the observed pl of a Pichia IFN with the reference material. While the IFN $a 2 b$ reference material showed the expected $\mathrm{pl}$ and was consistent with the theoretical value of 5.99 (ExPASy Computepl/MW), the Pichia sample showed a more acidic main band together with a faint extra acidic band. The nature of these modifications were then determined by LC-MS analysis.

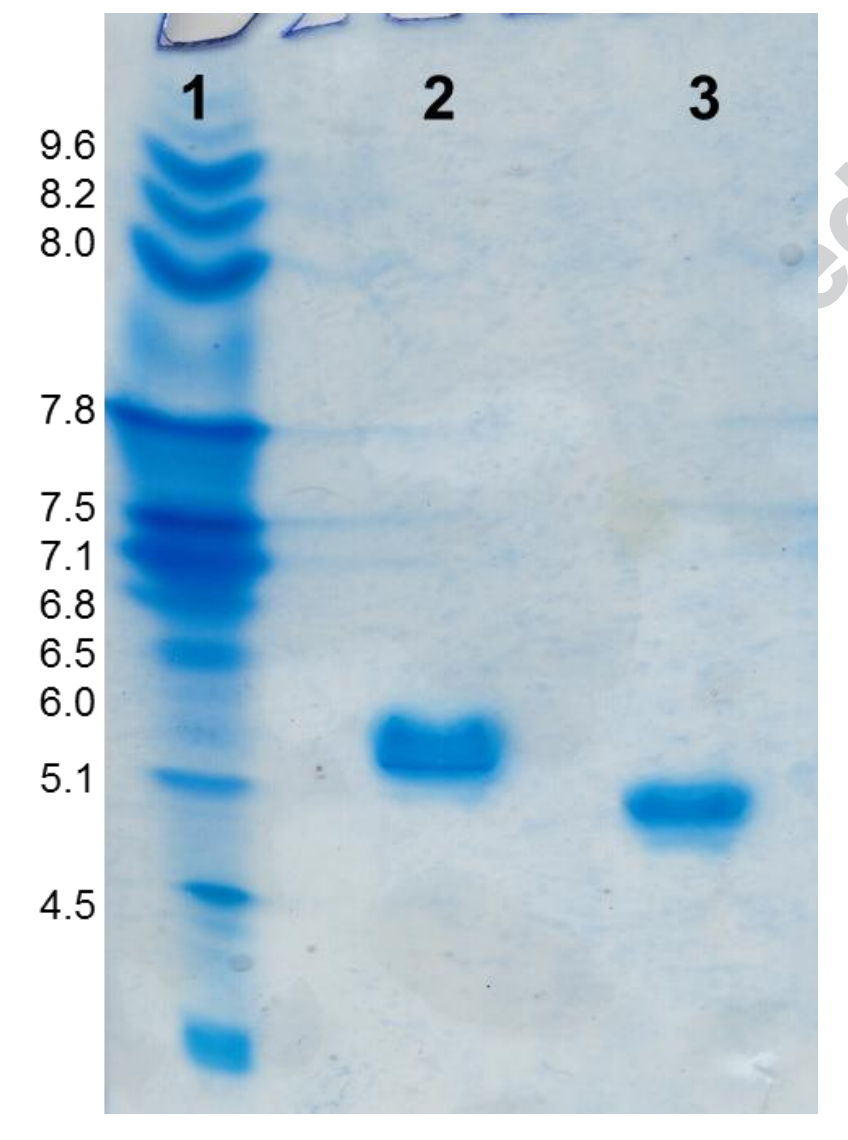

Figure 1: IEF gel of IFN $\alpha 2 b$ reference material and Pichia IFN $\alpha 2 b$. 
Lane 1: IEF standards, Lane 2: IFN $a 2 b$ reference material, Lane 3: Pichia IFN $a 2 b$ material.

\section{Trypsin digestion and LC/MS analysis of IFN $\alpha 2 b$ reference material and Pichia sample:}

\section{Determination of amino acid sequence:}

The goal of peptide mapping is to ensure that the primary structure of the drug substance is correct by obtaining $100 \%$ sequence coverage. Sometimes, one type of enzyme digestion will not give complete coverage due to factors such as limited cleavage sites in the protein with the generation of large peptides not suitable for LCMS analysis or peptides which are too short and polar to be retained on the HPLC column or too hydrophobic for elution. The peptides identified with trypsin digestion of IFN $a 2 b$ standard and Pichia IFN $a 2 b$ are listed in Table 1 . In this analysis, tryptic peptide T10 which contains residues 84 to 112 was not identified in the Pichia IFN analysis but was observed in the reference material. While the protein sequence for approved interferon $\alpha 2 b$ remains confidential, our LC/MS analysis confirmed the DrugBank database (https://www.drugbank.ca/) information which is at variance with the Uniprot (http://www.uniprot.org/) sequence information ( $N$ vs. $K$ at residue 112 respectively). We then confirmed that the sequence of the Pichia material was consistent with the sequence reported in Drugbank database by Glu-C digestion to generate a peptide length suitable for LC-MS analysis and that contains residue 112 as in TPLMNE (108-113) as in Figure 2. 


\begin{tabular}{|c|c|c|c|c|c|c|c|}
\hline Peptide \# & AA\# & Sequence & Observed $\mathrm{m} / \mathrm{z}$ & Theoretical m/z & Charge & ppm & $\begin{array}{c}\text { RT } \\
\text { (min) }\end{array}$ \\
\hline 1 & $1-12$ & CDLPQTHSLGSR & 685.826 & 685.828 & 2 & 2 & 26.4 \\
\hline 2 & $13-22$ & RTLMLLAQMR & 411.570 & 411.570 & 3 & 1 & 48.6 \\
\hline 3 & $14-22$ & TLMLLAQMR & 538.800 & 538.801 & 2 & 3 & 44.9 \\
\hline 4 & $23-31$ & RISLFSCLK & 562.318 & 562.318 & 2 & 0 & 52.7 \\
\hline 5 & $24-31$ & ISLFSCLK & 484.267 & 484.268 & 2 & 1 & 44.4 \\
\hline 6 & $32-49$ & DRHDFGFPQEEFGNQFQK & 1113.501 & 1113.504 & 2 & 2 & 47.7 \\
\hline 7 & $50-59$ & AETIPVLHEM & 570.290 & 570.292 & 2 & 3 & 39.2 \\
\hline 8 & $60-70$ & IQQIFNLFSTK & 669.875 & 669.875 & 2 & 1 & 50.0 \\
\hline 9 & $17-83$ & DSSAAWDETLLDK & 725.838 & 725.839 & 2 & 1 & 39.2 \\
\hline 10 & $84-112$ & FYTELYQQLNDLEACVIQGVGVTETPLMK & 1120.559 & 1120.556 & 3 & 3 & 63.9 \\
\hline 11 & $113-120$ & EDSILAVR & 451.751 & 451.751 & 2 & 1 & 30.6 \\
\hline 12 & $121-125$ & KYFQR & 371.205 & 371.206 & 2 & 2 & 25.9 \\
\hline 14 & $126-131$ & ITLYLK & 750.474 & 750.476 & 1 & 3 & 36.9 \\
\hline 15 & $132-133$ & EK & ND & 275.148 & 1 & NA & ND \\
\hline 16 & $134-144$ & KYSPCAWEVVR & 465.569 & 465.568 & 3 & 3 & 39.6 \\
\hline 17 & $145-149$ & AEIMR & 619.321 & 619.323 & 1 & 4 & 17.3 \\
\hline 18 & $150-162$ & SFSLSTNLQESLR & 741.382 & 741.383 & 2 & 1 & 42.4 \\
\hline 19 & $163-165$ & SKE & ND & 363.188 & 1 & NA & ND \\
\hline
\end{tabular}

Table 1: Peptide mapping result for IFN $\alpha 2 b$ standard using trypsin in-gel digestion.

${ }^{1}$ Several short peptides with two or three amino acids (T15, 2 amino acids) and T19, 3 amino acids), were too polar to retain on C18 column for LC-MS analysis. These missing amino acids were identified by Glu-C digestion to achieve $100 \%$ sequence coverage (data not shown). 

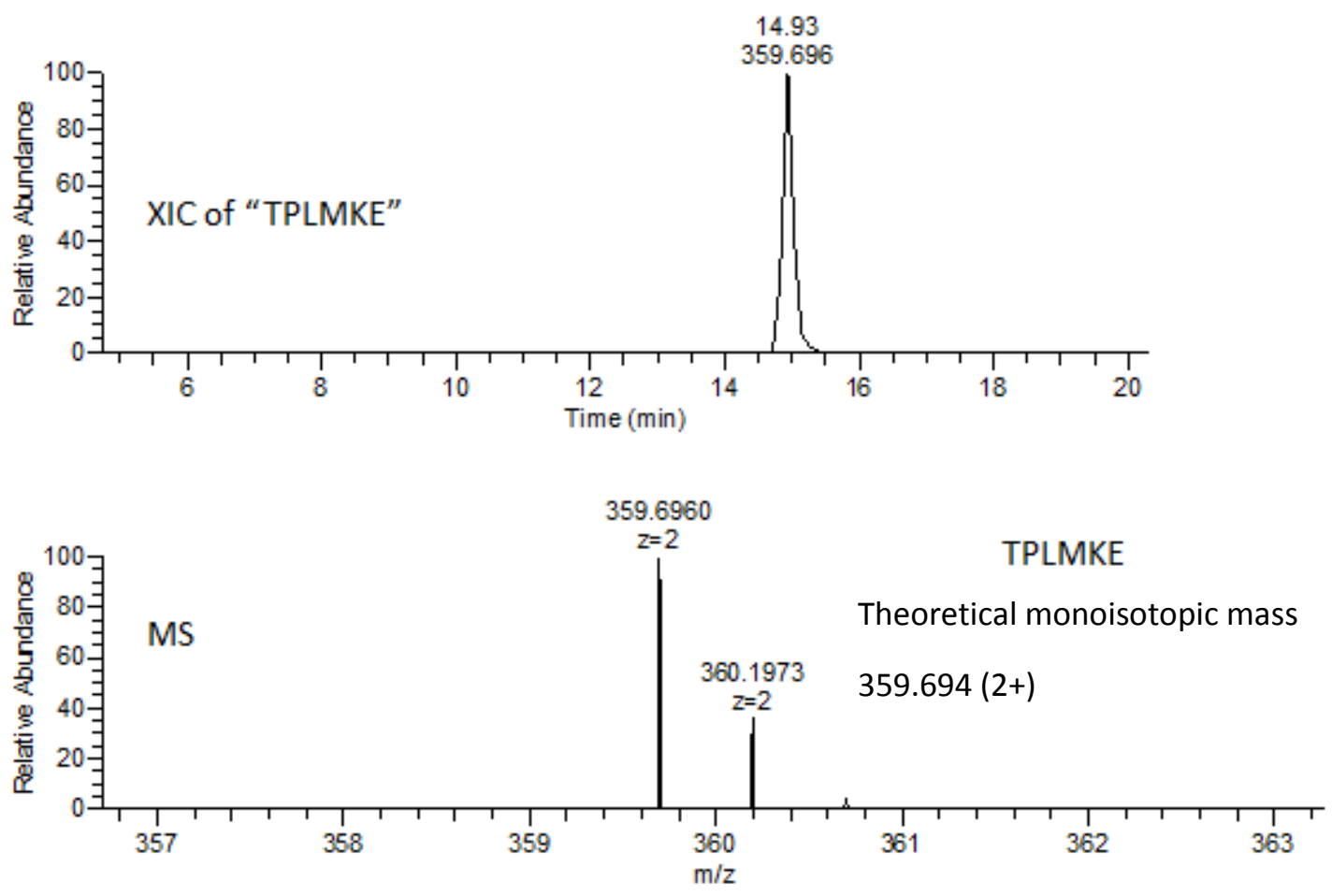

TPLMKE

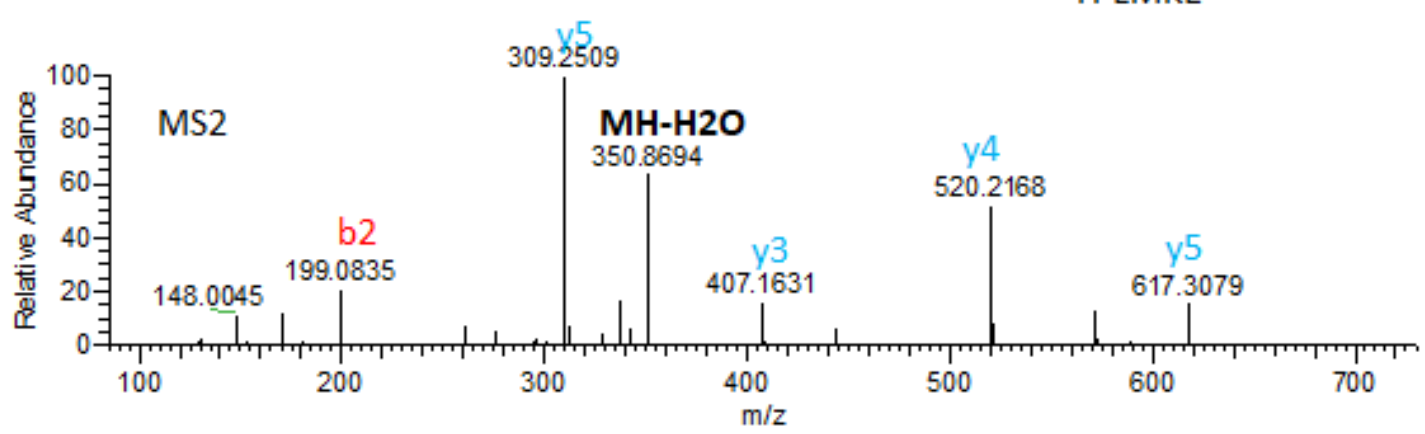

Figure 2: Confirmation of amino acid substitution K112 to N 112 of Pichia IFN a2b by GluC digestion.

A: Extracted Ion Chromatogram of "TPLMNE", B: Monoisotopic mass of "TPLMNE", C: CID MS2 of "TPLMNE".

Analysis of residual leader sequence: 
To examine if there is any residual leader sequence present in the process development samples we customized the expected protein sequence by combining the amino acid sequences for $\alpha$ factor and IFN $\alpha 2 b$. The trypsin digest LC/MS data was then searched against the combined FASTA file using Thermo Biopharma Finder 2.0. To effectively use Biopharma Finder for in-depth characterization of recombinant proteins there are several parameters critical for an effective database search. These parameters include $\mathrm{S} / \mathrm{N}$ ratio (set at default value 20 , unless the identification of a very low level of variant is needed), mass accuracy (set as $5 \mathrm{ppm}$ with a minimum confidence level of 0.8 to ensure low level of false positive identifications). With this approach, we determined that "EEGVSLEKR" was still attached to the $\mathrm{N}$-terminus of the Pichia IFN $a 2 b$ via the observation of the peptide "EEGVSLEK (see Figure S2) as well as "RCDLPQTHSLGSR" but these peptides were not present in the reference material (see Figure S3).

\section{Oxidation analysis}

The oxidation level comparison of reference IFN $\alpha 2 b$ and Pichia material was shown in Table 2. The percentage of oxidation was calculated using the following equation: peak area of oxidized peak / (peak area of oxidized peak + peak area of unoxidized peak) $\mathrm{x}$ $100 \%$. Except for the residue Met16 in Pichia material, which is oxidized at a slightly higher level than the standard material, the other four methionine residues have a similar oxidation level to the standard. The low level of oxidation observed with the reference material also indicates that our in-solution digestion protocol induced little artificial oxidation. 


\begin{tabular}{|c|c|c|}
\hline Site & Standard & Pichia \\
\hline Met 16 & $2.3 \%$ & $6.9 \%$ \\
\hline Met 21 & $2.9 \%$ & $1.9 \%$ \\
\hline Met 59 & $3.6 \%$ & $5.8 \%$ \\
\hline Met 111 & $1.0 \%$ & $2.6 \%$ \\
\hline Met 159 & $2.7 \%$ & $1.4 \%$ \\
\hline
\end{tabular}

Table 2: Oxidation analysis of IFN $\alpha 2 b$ reference material and Pichia material.

Part B: Analysis of Interferon (IFN) Y reference material (Abchem) and Pichia IFN Y

In-gel digestion of SDS-PAGE gel bands IFN $\mathrm{Y}$ with trypsin: N-glycosylation of Pichia IFN y

As stated above, for Pichia IFN y, glycosylation motif N-X-S/T, N98 (NYS) and N26 (NGT) present in the amino acid sequence can lead to a high level of glycosylation. The similarity of the amino acid sequence in the C-terminal and the signal peptide is the cause of C-terminal proteolysis, as the enzyme responsible for removal of signal peptide also recognize the amino acids near the C-terminus. Methionine and asparagine residues have the potential for oxidation and deamidation.

As shown in the gel image Figure S4, there were two bands observed for Pichia IFN fermentation supernatant (MW $17 \mathrm{KDA}$ and $19 \mathrm{KDA}$ ) and one band for the reference 
material (Abcam) at 15 KDA. LC-MS analysis of the in-gel trypsin digests of these bands was performed and the raw data was searched against the amino acid sequence of IFN $y$ together with that of the $\alpha$ factor (BioPharma Finder 2.0). For the 15 KDA reference material band, except a short peptide that has "KR" repeats, all of the expected sequences were identified. While in the 17 KDA Pichia IFN sample, all tryptic peptides were identified except the peptide containing residues 91-108 “DDFEKLTNYSVTDLNVQR" and the C-terminal peptide 127-144 “TGKRKRSQMLFRGRRASQ”. In the 19 KDA Pichia IFN y band, three peptides 15-35 "YFNAGHSDVADNGTLFLGILK", 91-108 "DDFEKLTNYS VTDLNVQR" and C-terminal peptide "TGKRKRSQMLFRGRRASQ" were not observed. After inspecting the amino acid sequence of these two missing peptides, we found each peptide contains an $\mathrm{N}$ glycosylation motif N-X-S/T, N98 (NYS) and N26 (NGT). In the 17 KDA band, N98 was fully glycosylated as shown in Figure 3 and N26 was not glycosylated. In the 19 KDA band, both N 26 (see Figure S5) and N 98 were fully glycosylated. N26 had mannosylation from Man9 extending to Man16 with Man 11 the highest glycoform. N98 contained mannosylation from Man6 to Man12 with Man9 as the highest abundance glycoform. Mannosylation was also the reason for the molecular weight shift that was observed on SDS-PAGE: the mass of glycan GalNAc2Man9 is around $1865 \mathrm{Da}$, which counts for the $\sim 2 \mathrm{KDA}$ shift for $17 \mathrm{KDA}$ band. The mass of glycan GalNAc2Man11 is around $2188 \mathrm{Da}$, which accounts for the additional 2 KDA mass shift for 19 KDA band. 


\begin{tabular}{|c|c|c|c|c|c|c|c|}
\hline$\#$ & AA\# & Sequence & Observed $\mathrm{m} / \mathrm{z}$ & Theoretical $\mathrm{m} / \mathrm{z}$ & charge & ppm & $\mathrm{RT}(\min )$ \\
\hline 1 & $1-14$ & MQDPYVKEAENLKK & 564.961 & 564.959 & 3 & 3 & 24.33 \\
\hline 2 & $15-35$ & YFNAGHSDVADNGTLFLGILK & 1126.572 & 1126.571 & 2 & 1 & 42.83 \\
\hline 3 & $36-44$ & NWKEESDRK & 397.864 & 397.863 & 3 & 2 & 17.18 \\
\hline 4 & $45-53$ & IMQSQIVSF & 526.777 & 526.776 & 2 & 2 & 30.24 \\
\hline 5 & $54-56$ & YFK & 457.245 & 457.245 & 1 & 1 & 16.52 \\
\hline 6 & $57-69$ & LFKNFKDDQSIQK & 537.625 & 537.623 & 3 & 4 & 23.25 \\
\hline 7 & $70-81$ & SVETIKEDMNVK & 696.857 & 696.856 & 2 & 2 & 19.46 \\
\hline 8 & $82-88$ & FFDSNKK & 443.228 & 443.227 & 2 & 2 & 18.20 \\
\hline 9 & $89-95$ & KRDDFEK & 313.163 & 313.163 & 3 & 1 & 16.45 \\
\hline 10 & $96-108$ & LTNYSVTDLNVQR & 761.898 & 761.897 & 2 & 2 & 29.53 \\
\hline 11 & $109-118$ & KAIHELIQVM & 591.340 & 591.339 & 2 & 1 & 31.10 \\
\hline 12 & $119-126$ & AELSPAAK & 786.436 & 786.436 & 1 & 1 & 17.57 \\
\hline 13 & $127-144$ & TGKRKR & ND & 745.479 & 1 & NA & ND \\
\hline 14 & $133-144$ & SQMLFRGRRASQ & 718.879 & 718.881 & 2 & -2 & 27.23 \\
\hline
\end{tabular}

Table 3: Peptide mapping of IFN y reference material with trypsin digestion.

Except 127-132 "TGKRKR" that has the KRKR repeats, all the peptides were covered by trypsin digestion. 

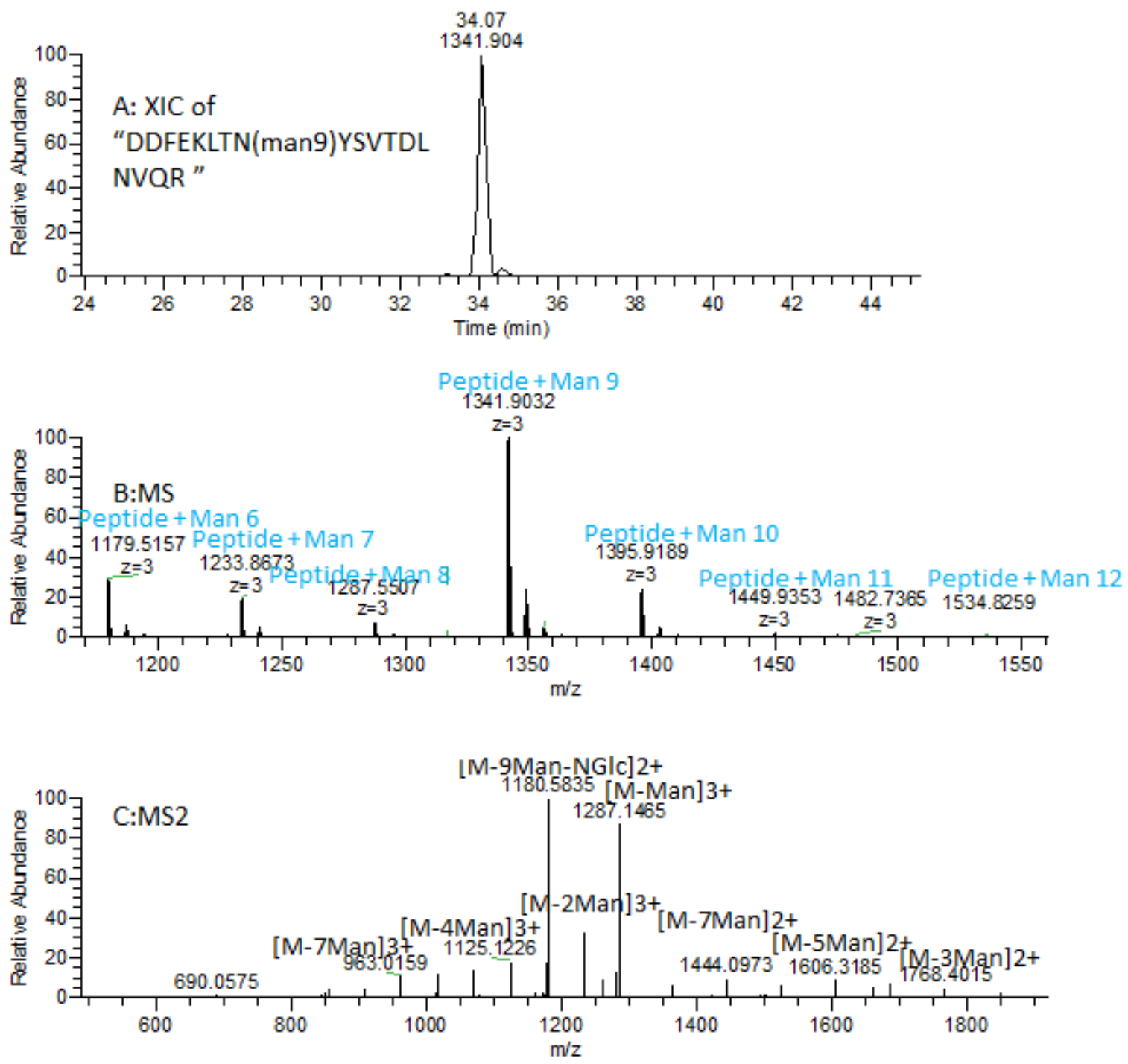

Figure 3: Identification of Man9 glycosylation at N98 in the 17 KDA band.

A: Extracted Ion Chromatogram of "DDFEKLTN (man9) YSVTDLNVQR" B:

Monoisotopic mass of mannosylation from Man6 to Man12, all the glycoforms coelute.

C: CIDMS2 of the highest glycoform Man9.

\section{Oxidation and deamidation}


The analysis of crude IFN $\mathrm{Y}$ process development sample requires an in-gel digestion analysis which can induce high levels of degradation products during the extensive sample preparation steps. The IFN $\mathrm{y}$ amino acid sequence contains two deamidation hot-spots, NG of N26 and NS of N84. In the 17 KDA band, the deamidation level of N26 is $>90 \%$ and N84 is $20 \%$. In the 19 KDA band, as N26 is fully glycosylated, no deamidation was observed in that site. The observed amounts of oxidation and deamidation level are summarized in Table 4.

\begin{tabular}{|c|c|c|c|c|}
\hline Sites & Modification & Reference & Pichia 17KDa & Pichia 19KDa \\
\hline Met1 & Oxidation & $76.6 \%$ & $87.8 \%$ & $72.0 \%$ \\
\hline Met46 & Oxidation & $81.0 \%$ & $85.4 \%$ & $77.2 \%$ \\
\hline Met78 & Oxidation & $67.6 \%$ & $72.4 \%$ & $72.6 \%$ \\
\hline Met118 & Oxidation & $22.2 \%$ & $38.5 \%$ & $21.7 \%$ \\
\hline Asn26 & Deamidation & $>90 \%$ & $>90 \%$ & NA \\
\hline Asn84 & Deamidation & $5.2 \%$ & $22.7 \%$ & $6.5 \%$ \\
\hline
\end{tabular}

Table 4: Oxidation analysis of IFN y reference material and Pichia material. FOOTNOTE NA = Not available

\section{In-gel digestion of IFN y SDS-PAGE gel bands with Glu-C: C-terminal proteolysis}

GluC digestion was performed on the two bands of lane 15 in the SDS-PAGE gel as well as the reference material (Lane 1). As there are only a few glutamic acid residues ("E") in the IFN Y sequence, the observed sequence coverage by Glu-C digestion was low. However, residues121-129 "LSPAAKTGK" was identified in the Pichia IFN y but not 
the reference material indicating the proteolysis had occurred at residue $129(\mathrm{~K})$ in the Pichia material (Figure S6).

\section{Discussion:}

Characterization of process development material at an early stage is a challenging yet important task because early analytical information can accelerate the development process. The analytical challenges include low concentrations of the product in fermentation samples, high level of interferences caused by media components and host cell proteins (HCPs) as well as unknown modifications in the product molecule. Recently, mass spectrometry based methods have enjoyed a dramatic growth in popularity, as they offer a variety approaches to efficiently gain diverse information on product quality. For biosimilar development, there is both the need for efficient process development material and a suitable reference material. For the approval process, it is necessary to characterize the protein amino acid sequence at a $100 \%$ level as well as any post-translational modifications and degradation products. Since any structural changes can have effects on drug potency and have safety effects such as generation of immune responses in a patient, ${ }^{16}$ it is necessary to have analytical methods for complete product characterization.

As discussed previously, Pichia pastoris has gained wide application for protein production with several successful cases of recombinant therapeutic protein production in yeast which demonstrated its unique features such as low cost, high growth rate, usage of defined media and low level of secretion of HCPs. ${ }^{17}$ For example, Biocon, a Indian biotechnology company, implement its Pichia pastoris platform for the production of insulin and insulin analogs and successfully commercialized the large-scale 
manufacture of human insulin. Common characteristics of protein variants expressed in Pichia pastoris include residual leader sequence when using an a factor signal peptide and high mannose $\mathrm{N}$-glycosylation structures. The complex glycosylation patterns can also impede the pharmaceutical development process and hyper mannosylation and

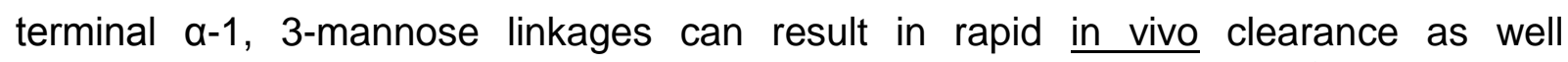
immunogenicity of the recombinant protein in clinical studies. ${ }^{18-20}$

Since interferon $a 2 b$ and interferon $y$ have distinctly different amino acid sequences therefore can contain a range of product variants at various sites. Thus, multiple analytical strategies are required for the in-depth characterization of all the potential variants. As summarized in Tables 5 and 6 , the variants of these two IFN product require the design of different LC/MS analytical methods where it is important to optimize the selection of the appropriate protease to generate peptides containing the variant sites that are appropriate for LC-MS analysis. In the case of the IFNY process development, the need for the analysis of crude fermentation samples added an additional analytical challenge. In this case, we demonstrated that in-gel digestion rather than in-solution digestion is the method of choice. The SDS-PAGE approach used in this analysis has the following advantages: removal of any detergents present in the sample that can be detrimental to mass spectrometric analysis, the target protein can be separated from most HCPs, proteolysis and covalent aggregation can be observed with reduced and non-reduced gels ahead of MS analysis. One drawback of the in-gel digestion method is the high level of oxidation artifacts that can be induced during the sample preparation process. On the other hand, in-solution enzyme digestion which is 
suitable for purified material as the case of IFN $\alpha 2 b$ can greatly reduce the level of artifacts.

\begin{tabular}{|c|c|c|c|}
\hline \multicolumn{4}{|c}{ Interferon $\mathbf{\alpha} \mathbf{2 b}$} \\
\hline residue & variants & peptides & enzyme \\
\hline EEGVSLEKR & Leader sequence & $\begin{array}{c}\text { EEGVSLEK and } \\
\text { RCDLPQTHSLGSR }\end{array}$ & trypsin \\
\hline $112 \mathrm{~N}$ & Amino acid substitution & $118-123:$ TPLMNE & Glu-C \\
\hline 16 Met & Oxidation & $14-22:$ TLMLLAQMR & trypsin \\
\hline 21 Met & Oxidation & $14-22:$ TLMLLAQMR & trypsin \\
\hline 59 Met & Oxidation & $50-59:$ AETIPVLHEM & trypsin \\
\hline 111 Met & Oxidation & $118-123:$ TPLMNE & Glu-C \\
\hline 159 Met & Oxidation & $145-149:$ AEMIR & trypsin \\
\hline
\end{tabular}

Table 5: Variants identified for Pichia IFN a2b. 


\begin{tabular}{|c|c|c|c|}
\hline \multicolumn{4}{|c|}{ Interferon V } \\
\hline residue & variants & peptides & enzyme \\
\hline 26 Asn & Glycosylation & 15-35: YFNAGHSDVADNGTLFLGILK & trypsin \\
\hline 98 Asn & Glycosylation & $96-108:$ LTNYSVTDLNVQR & trypsin \\
\hline $129 \mathrm{~K}$ & Proteolysis & 121-129: LSPAAKTGK & Glu-C \\
\hline 1 Met & Oxidation & $1-7:$ MQDPYVK & trypsin \\
\hline $46 \mathrm{Met}$ & Oxidation & $45-53:$ IMQSQIVSF & trypsin \\
\hline $78 \mathrm{Met}$ & Oxidation & SVETIKEDMNVK & trypsin \\
\hline $118 \mathrm{Met}$ & Oxidation & 109-118: KAIHELIQVM & trypsin \\
\hline 26 Asn & Deamidation & $15-35:$ YFNAGHSDVADNGTLFLGILK & trypsin \\
\hline 84 Asn & Deamidaiton & $82-87:$ FFNSNK & trypsin \\
\hline
\end{tabular}

\section{Table 6: Variants identified for Pichia IFNy.}

The above HPLC/MS analyses generated with our multi-enzyme digest strategy was developed for the characterization of IFNs $\alpha 2 b$ and $y$ in process development samples. For Pichia IFN $a 2 b$ the major variants that were observed were an amino acid substitution and residual leader sequence. Amino acid substitutions can occur by clonal variation in the host cell line ${ }^{21}$ or the use of a different cDNA sequence used in cell line construction $^{22}$. Such changes may obviously have effects on protein structure and function $^{23}$ and typically can only be detected via a complete determination of the amino acid sequence, but in the case of a charged to neutral amino acid substitution such a variant can be readily screened by isoelectric focusing (IEF). In this example, the variant was a substitution of lysine to asparagine (K112N, pKa10.6, reference material) which results in the acidic shift observed in the IEF gel (see Figure 1). In this manner, an IEF gel can be used as a quick assessment of product variants with pKa differences that can be then used to assess process development samples. 
. Every aspect of the protein structure including amino acid sequence, post-translational modifications (PTMs) is related to the characteristics of a process, including strain construction, cultivation conditions and downstream purification. The quality of early stage process development material reflects the efficacy of the manufacturing platform and an early stage analytical evaluation is critical for host system selection, media optimization and cultivation conditions. For example, a high level of oxidation in the product may be due to a high oxygen density in bioreactor, proteolysis characterization can be beneficial for selection of a suitable protease inhibitor, aggregation characterization is useful for the optimization of $\mathrm{pH}$ in the fermentation and suggest the need for addition of detergents to the formulation buffer. ${ }^{24}$

\section{Conclusion:}

A multi-enzyme digestion approach was successfully developed for the characterization of interferon products expressed from Pichia pastoris. For IFN $\alpha 2 b$, trypsin digestion was used initially for peptide mapping, identification of leader sequence and amino acid substitution. Glu-C digestion also provided complementary data for the characterization of an amino acid substitution. An IEF gel method followed by LC/MS analysis was developed as a quick way to monitor the acidic shift of pl due to an amino acid substitution.

In-gel digestion with trypsin or Glu-C was developed for the characterization of a crude, unpurified IFN $\mathrm{Y}$ strain supernatant. The material was shown to have mannosylation at the two N-glycosylation sites and C-terminal proteolysis. Pichia pastoris has gain popularity and is attractive to the biotherapeutic industry due to its rapid growth in culture and efficient protein secretion. The method developed above could be extremely 
helpful especially for process development at an early stage where prompt analytical feedback can help optimize strain development and fermentation conditions in Pichia pastoris strains.

\section{Acknowledgement}

This work was supported by the Defense Advanced Research Projects Agency (DARPA) and SPAWAR Systems Center Pacific (SSC Pacific) under Contract No. N66001-13-C-4025. The content is solely the responsibility of the authors and does not necessarily represent the official views of DARPA or SSC.

\section{Author Information}

* Corresponding Author

wi.hancock@northeastern.edu

Notes

The author declares no competing financial interest. And no patients or animals are involved in this research.

\section{Reference:}

(1) Asmana Ningrum, R., Human interferon alpha-2b: a therapeutic protein for cancer treatment. Scientifica (Cairo) 2014, 2014, 970315.

(2) Parker, B. S.; Rautela, J.; Hertzog, P. J., Antitumour actions of interferons: implications for cancer therapy. Nat Rev Cancer 2016, 16 (3), 131-44.

(3) Liu, Y. H.; Wylie, D.; Zhao, J.; Cure, R.; Cutler, C.; Cannon-Carlson, S.; Yang, X.; Nagabhushan, T. L.; Pramanik, B. N., Mass spectrometric characterization of the isoforms in Escherichia coli recombinant DNA-derived interferon alpha-2b. Anal Biochem 2011, 408 (1), 105-17. 
(4) Schroder, K.; Hertzog, P. J.; Ravasi, T.; Hume, D. A., Interferon-gamma: an overview of signals, mechanisms and functions. J Leukoc Biol 2004, 75 (2), 163-89.

(5) https://adisinsight.springer.com/drugs/800038646

(6) Ahmad, M.; Hirz, M.; Pichler, H.; Schwab, H., Protein expression in Pichia pastoris: recent achievements and perspectives for heterologous protein production. Appl Microbiol Biotechnol 2014, 98 (12), 5301-17.

(7) Waters, M. G.; Evans, E. A.; Blobel, G., Prepro-alpha-factor has a cleavable signal sequence. J Biol Chem 1988, 263 (13), 6209-14.

(8) Cereghino, J. L.; Cregg, J. M., Heterologous protein expression in the methylotrophic yeast Pichia pastoris. FEMS Microbiol Rev 2000, 24 (1), 45-66.

(9) He, Z.; Huang, Y.; Qin, Y.; Liu, Z.; Mo, D.; Cong, P.; Chen, Y., Comparison of alphafactor preprosequence and a classical mammalian signal peptide for secretion of recombinant xylanase xynB from yeast Pichia pastoris. $J$ Microbiol Biotechnol 2012, 22 (4), 479-83.

(10) Fitzgerald, I.; Glick, B. S., Secretion of a foreign protein from budding yeasts is enhanced by cotranslational translocation and by suppression of vacuolar targeting. Microb Cell Fact 2014, 13 (1), 125.

(11) Gemmill, T. R.; Trimble, R. B., Overview of N- and O-linked oligosaccharide structures found in various yeast species. Biochim Biophys Acta 1999, 1426 (2), 227 37.

(12) Hamilton, S. R.; Davidson, R. C.; Sethuraman, N.; Nett, J. H.; Jiang, Y.; Rios, S.; Bobrowicz, P.; Stadheim, T. A.; Li, H.; Choi, B. K.; Hopkins, D.; Wischnewski, H.; Roser, J.; Mitchell, T.; Strawbridge, R. R.; Hoopes, J.; Wildt, S.; Gerngross, T. U., 
Humanization of yeast to produce complex terminally sialylated glycoproteins. Science 2006, 313 (5792), 1441-3.

(13) Wang, D.; Ren, H.; Xu, J. W.; Sun, P. D.; Fang, X. D., Expression, purification and characterization of human interferon- $y$ in Pichia pastoris. Mol Med Rep 2014, 9 (2), 7159.

(14) Choudhary, G.; Wu, S. L.; Shieh, P.; Hancock, W. S., Multiple enzymatic digestion for enhanced sequence coverage of proteins in complex proteomic mixtures using capillary LC with ion trap MS/MS. J Proteome Res 2003, 2 (1), 59-67.

(15) Wang, Y. A.; Wu, D.; Auclair, J. R.; Salisbury, J. P.; Sarin, R.; Tang, Y.; Mozdzierz, N. J.; Shah, K.; Zhang, A. F.; Wu, S. L.; Agar, J. N.; Love, J. C.; Love, K. R.; Hancock, W. S., Integrated Bottom-Up and Top-Down Liquid Chromatography-Mass Spectrometry for Characterization of Recombinant Human Growth Hormone Degradation Products. Anal Chem 2017, 89 (23), 12771-12777.

(16) Moussa, E. M.; Panchal, J. P.; Moorthy, B. S.; Blum, J. S.; Joubert, M. K.; Narhi, L. O.; Topp, E. M., Immunogenicity of Therapeutic Protein Aggregates. $J$ Pharm Sci 2016, 105 (2), 417-430.

(17) Bollok, M.; Resina, D.; Valero, F.; Ferrer, P., Recent patents on the Pichia pastoris expression system: expanding the toolbox for recombinant protein production. Recent Pat Biotechnol 2009, 3 (3), 192-201.

(18) Kalidas, C.; Joshi, L.; Batt, C., Characterization of glycosylated variants of betalactoglobulin expressed in Pichia pastoris. Protein Eng 2001, 14 (3), 201-7. (19) Teh, S. H.; Fong, M. Y.; Mohamed, Z., Expression and analysis of the glycosylation properties 
of recombinant human erythropoietin expressed in Pichia pastoris. Genet $\mathrm{Mol}$ Biol 2011, 34 (3), 464-70.

(20) Dai, M.; Yu, C.; Fang, T.; Fu, L.; Wang, J.; Zhang, J.; Ren, J.; Xu, J.; Zhang, X.; Chen, W., Identification and Functional Characterization of Glycosylation of Recombinant Human Platelet-Derived Growth Factor-BB in Pichia pastoris. PLoS One 2015, 10 (12), e0145419.

(21) Asare, E.; Mugavero, J.; Jiang, P.; Wimmer, E.; Paul, A. V., A Single Amino Acid Substitution in Poliovirus Nonstructural Protein 2CATPase Causes Conditional Defects in Encapsidation and Uncoating. J Virol 2016, 90 (14), 6174-6186.

(22) Ruiz-Jarabo, C. M.; Pariente, N.; Baranowski, E.; Dávila, M.; Gómez-Mariano, G.; Domingo, E., Expansion of host-cell tropism of foot-and-mouth disease virus despite replication in a constant environment. J Gen Virol 2004, 85 (Pt 8), 2289-97.

(23) Ng, P. C.; Henikoff, S., Predicting the effects of amino acid substitutions on protein function. Annu Rev Genomics Hum Genet 2006, 7, 61-80.

(24) Crowell, L. E.; Lu, A. E.; Love, K. R.; Stockdale, A.; Timmick, S. M.; Wu, D.; Wang, Y. A.; Doherty, W.; Bonnyman, A.; Vecchiarello, N.; Goodwine, C.; Bradbury, L.; Brady, J. R.; Clark, J. J.; Colant, N. A.; Cvetkovic, A.; Dalvie, N. C.; Liu, D.; Liu, Y.; Mascarenhas, C. A.; Matthews, C. B.; Mozdzierz, N. J.; Shah, K. A.; Wu, S. L.; Hancock, W. S.; Braatz, R. D.; Cramer, S. M.; Love, J. C., On-demand manufacturing of clinical-quality biopharmaceuticals. Nat Biotechnol 2018. 36, 988-995. 